\title{
To investigate the clinical effect of modified closed negative pressure suction technique combined with flap transplantation on the treatment of deep chronic refractory wounds
}

\author{
Zhihui Hou ${ }^{1}$, Mingjuan $\mathrm{Gu}^{* 2}$ \\ ${ }^{1}$ Department of Burns, Baogang Hospital, Baotou, Inner Mongolia, China \\ ${ }^{2}$ Department of Plastic Surgery, Baogang Hospital, Baotou, Inner Mongolia, China
}

Received: January 25, 2019

DOI: $10.5430 /$ dcc.v6n1p11
Accepted: March 2, 2019

Online Published: March 10, 2019

\begin{abstract}
Objective: To investigate the clinical effect of modified closed negative pressure suction technique combined with flap transplantation on the treatment of deep chronic refractory wounds.

Methods: During March of 2015 to April of 2018, 52 cases of patients with deep chronic refractory wounds were selected as research objects. They were divided into the control group and the treatment group by use of the random number table method, with 26 cases in each group. Among them, the control group was given conventional debridement combined with flap reconstruction, and the treatment group was treated with modified closed negative pressure suction technique combined with flap transplantation to observe the clinical effect.

Results: (1) According to the analysis on the effect of flap transplantation, the excellent and good rate of the treatment group was 92.3\%, and in the control group, it was $76.9 \%(p<.05)$. (2) According to the statistics, the incidence of complications in the treatment group was lower than that in the control group $(p<.05)$.

Conclusions: Modified closed negative pressure suction technique combined with flap transplantation has a good effect on the treatment of deep chronic refractory wounds with fewer complications.
\end{abstract}

Key Words: Modified closed negative pressure suction technique, Flap transplantation, Deep chronic refractory wounds

\section{INTRODUCTION}

Deep chronic refractory wounds are a special type of wounds, the main characteristics of which are impaired local blood circulation in the wound, lack of angiogenesis, fibrogenesis and cicatrices in the inflammatory wound tissues, subcutaneous tissue atrophy, leading to refractory wounds. ${ }^{[1]}$ With respect to flap transplantation, flaps can be divided into flat flaps and tubed flaps according to flap morphology, and they also can be divided into local flaps and distant flaps according to flap pattern and the repair of skin defects. After the 1970s, a classification method based on the type of flap blood circulation was proposed in academic circles, i.e., random pattern skin flaps and axial pattern skin flaps. ${ }^{[2]}$ Modified closed negative pressure suction technique is a new method

\footnotetext{
*Correspondence: Mingjuan Gu; Email: nmgbgyyyy@163.com; Address: Department of Plastic Surgery, Baogang Hospital, Baotou, Inner
} Mongolia, 014010, China. 
which can improve blood perfusion and oxygen supply in the wound, optimize blood circulation, kill bacteria and control exudation of wound surface. It can promote fibroblast growth and accelerate wound healing through wound moisture and physical retraction. ${ }^{[3]}$ The research is designed to investigate the effect of modified closed negative pressure suction technique combined with flap transplantation on the treatment of deep chronic refractory wounds.

\section{DATA AND METHODS}

\subsection{Basic data}

During March of 2015 to April of 2018, 52 cases of patients with deep chronic refractory wounds were selected as research objects. They were divided into the control group and the treatment group by use of the random number table method, with 26 cases in each group. There were 15 male patients and 11 female patients in the control group, aged $43-75$, with the average age of $(61.3 \pm 5.82)$. The course of disease was 2-11 weeks, with the average of $(6.1 \pm 0.37)$ weeks. The area of wound was $11-17 \mathrm{~cm}^{2}$, with the average of $(14.5 \pm 1.37) \mathrm{cm}^{2}$. Among them, there were 11 cases of skin defects on the leg, 8 cases on the face, 5 cases on the arm and 2 cases on the ankle. There were 16 male patients and 10 female patients in the treatment group, aged 40-76, with the average of $(60.9 \pm 6.43)$. The course of disease was 3-9 weeks, with the average of $(5.9 \pm 0.53)$ weeks. The area of wound was $11-18 \mathrm{~cm}^{2}$, with the average of $(15.1 \pm$ 1.07) $\mathrm{cm}^{2}$. Among them, there were 10 cases of skin defects on the leg, 10 cases on the face, 5 cases on the arm and 1 case on the ankle. It was of comparability as there was no statistically significant difference in the comparison of basic data between the two groups of patients $(p>.05)$.

\subsection{Methods}

After admission, two groups of patients were given conventional treatment, such as supportive therapy, the improvement of local blood circulation, thorough debridement and so on.

The control group was given conventional debridement combined with flap reconstruction. The procedures were illustrated as follows: the surface of wound was covered by iodophor-vaseline gauze, and bound up with sterile gauzes and bandages. Once the surface of wound got infected again, or necrotic tissues appeared, it ought to perform a surgical debridement. If there were no necrotic tissues in the wound and the granulation tissues grew well, it was practicable to choose a suitable flap for the reconstruction. After surgery, it was required to give routine dressing change one or two times a day.

The treatment group was given modified closed negative pressure suction technique combined with flap transplantation, and the procedures were listed below: the surface of wound was covered by Vacuum sealing drainage (VSD) dressing, and one or several $10 \#$ or $12 \#$ silica gel collapsible bulbs were threaded through the deep wound or the necrotic area of bone infection, and fixed to the bottom of VSD dressing. Simple interrupted suture was performed to manage the incised edge of skin and VSD dressing. $75 \%$ ethanol was used to wipe the edge of the skin, and the wound was completely sealed by biological permeable films. Whereas, VSD drainage tube was connected to a three-way valve to give negative pressure drainage. If the dressing showed orange peel-like appearance, it indicated that the vacuum device was in a good state with no leakage. Besides, the negative pressure was kept in the range of $70-80 \mathrm{mmHg}$. After surgery, the silica gel collapsible bulbs were continuously washed by a large number of normal saline, and then filled with $20 \mathrm{ml}$ of rb-bFGF two times a day. During the process of filling, it was required to cease negative pressure drainage and the washing procedure of normal saline, which were not started until 15 minutes after the end of the filling process. After 7-10 days, granulation tissues grew well. At that time, VSD dressing was removed in order to choose a suitable flap for reconstruction.

\subsubsection{Indicator observation and effective analysis}

(1) Evaluation of the effect of flap transplantation: A.Excellent: No redness and swelling, the edge of flap matched well to the wound skin. B. Good: The edge between the flap and the wound skin cracked, and was almost healed after negative pressure suction and secondary suture. C. Poor: Necrosis appeared on the edge between the flap and the wound skin, with cracking, purulent secretion, the wound unhealed. ${ }^{[4]}$

(2) Statistics of complications in two groups of patients.

\subsubsection{Statistical methods}

The data used in this research were entered in the EXCEL form, SPSS20.0 software was applied to statistical analysis, and categorical data were represented by percentage $(\%)$, with $\chi^{2}$ test used. The difference $p<.05$ was of statistical significance.

\section{RESULTS}

\subsection{Effect of flap transplantation}

In the treatment group of 26 patients, there were 15 cases of patients with excellent effect, 9 cases with good effect and 2 cases with poor effect, and the excellent and good rate was $92.3 \%$ (24/26); in the control group of 26 patients, there were 7 cases of patients with excellent effect, 13 cases with good effect and 6 cases with poor effect, and the excellent and good rate was $76.9 \%(20 / 26)$. There was statistically 
significant difference in the comparison between two groups $\left(\chi^{2}=9.101, p<.05\right)$.

\subsection{Complications}

According to statistics, 1 case in the treatment group showed lacuna under the flap, and the incidence rate of complications was $3.8 \%$ (1/26); 2 cases in the control group showed lacuna under the flap, 1 case of anemia, 1 case of hypoproteinemia, and the incidence rate of complications was $15.3 \%$ (4/26). There was statistically significant difference in the comparison between the two groups $\left(\chi^{2}=7.655, p<.05\right)$.

\section{Discussion}

Deep chronic refractory wound, with a complicated pathogenesis, is commonly seen in patients with radiotherapy, senior diseases and diabetes mellitus. Even though refractory chronic ulcer will not produce a threat to the patients' life immediately, wound disunion of long time may lead to the loss of proteins in a large amount, reduce physical fitness and cause primary and basic diseases. Once the infection spreads, various complications such as sepsis will happen and even lead to death. ${ }^{[5]}$ Therefore, once a patient is diagnosed as deep chronic refractory wound clinically, it is needed to analyze the patient's condition and give the symptomatic treatment. Modified closed negative pressure suction technique, to some extent, avoids the infection caused by the exposure of wound after thorough debridement. To perform flap transplantation during the period of negative pressure suction, can intensify the covering and protection to the deep tissues, keep an abundant blood circulation, enhance the antiinfective ability and reconstruct the function and appearance of the impaired area. ${ }^{[6]}$ It is worthy to be noted that thorough debridement is the basis and precondition of good flap reconstruction. The survival rate of flap can be improved only by the thorough debridement of inflammatory granulation tissues and peripheral necrotic tissues. ${ }^{[7]}$

VSD refers to a technique to perform negative pressure suction by use of VSD materials \& semipermeable membrane $\&$ three-way valve \& vacuum suction device. ${ }^{[8]}$ Wim Fleischmann, M.D., from the affiliated Trauma Hospital of Ulm University in Germany, first pioneered VSD technique to be applied to the treatment of chronic refractory wound. ${ }^{[9]}$ VSD technique can relieve tissue edema of the wound, prevent the pressure from further impairing the tissues, improve the microcirculation, control oxygen consumption, reconstruct part of potentially damaged tissues, improve the expression of the markers for vascular endothelial growth factors and vascular endothelial cells (CD34), increase the proliferation and activity of fibroblasts and vascular endothelial cells in the wound, increase the density of capillaries, contributing to

Published by Sciedu Press the wound healing. ${ }^{[10]}$ The therapeutic mechanism of VSD technique is as follows: on the basis of the closed negative pressure condition, this technique is intended to prevent bacteria from invading the wounds, timely expel liquefied or necrotic metabolic wastes, reduce toxin absorption, improve the condition of the whole body, depress bacterial accumulation or reproduction, decrease wound infection and promote wound healing. ${ }^{[11]}$ In the clinical application of VSD technique, to ensure the clinical effect, it is necessary to enhance wound nursing management: (1) Continuous and effective negative pressure on the wound is the key to the success of drainage and treatment. Therefore, in the nursing management, it is needed to maintain a suitable pressure, keep an unblocked and tightly connected tube and properly fix the drainage tube. When the drainage is not smooth, $20 \mathrm{ml}$ of syringe is used to extract the wastes or $10-20 \mathrm{ml}$ of $0.9 \%$ normal saline is used to wash the tube. Replace the drainage tube if necessary. (2) Patients are suggested to eat highcalorie, high-vitamin and light food, in order to promote the growth of granulation tissues in the wound and prevent the complications. ${ }^{[12]}$

In this research, the control group was given conventional debridement combined with flap reconstruction, and the treatment group was treated with modified closed negative pressure suction technique combined with flap transplantation. The results showed that the excellent and good rate of flap transplantation in the treatment group was higher than that in the control group $(92.3 \%$ vs. $76.9 \%, p=.002)$, and the incidence of complications in the treatment group was lower than that in the control group (3.8\% vs. $15.3 \%, p=.005)$. The research also showed that VSD technique is of high value in clinical application. However, in the practical operation, it should be noted that: (1) Thorough debridement of necrotic tissues and foreign matters in the wound is guaranteed; (2) Under the aseptic condition, the used high polymer material is trimmed according to the size and characteristics of the wound, multiple pieces of materials can be used when the size of the wound is large. Besides, it is necessary to make the polymer material sufficiently contact with the surface of wound. (3) All side holes and the top part of the drainage tube should be embedded in the polymer material. (4) The surface of wound is tightly sealed. (5) The vacuum suction device can be connected to the negative pressure of the drainage tube. (6) Once the wound is cleaned, it is feasible to perform a secondary suture, free skin graft or tissue flap transplantation. (7) Combine with the anti-infective treatment if necessary. (8) High negative pressure drainage may cause bleeding, so that it is required to achieve a thorough haemostasis and keep away from blood vessels when debriding the wound. It is also suggested to pay close attention to 
the bleeding condition. The symptomatic treatment should be given if necessary.

\section{Conclusions}

In conclusion, modified closed negative pressure suction technique combined with flap transplantation has a satisfac- tory effect on the treatment of deep chronic refractory wound, and it is suggested to be applied clinically.

\section{CONFlicts OF INTEREST Disclosure}

The authors declare they have no conflicts of interest.

\section{REFERENCES}

[1] Yan Z, Xiaofeng Y, Guoxia L, et al. The clinical curative effect of modified closed suction technique combined with flap transplantation in treating chronic refractory wound depth. Clinical Journal of Chinese Medicine. 2017; 9(06): 109-110.

[2] Xincheng L, Guanghua G. Classification and clinical evaluation of chronic refractory wounds. Chinese Journal of Injury Repair and Wound Healing (Electronic Edition). 2017; 12(04): 303-305.

[3] Zhiyong W, Xiaopei H, Xin X, et al. Research progress of the pathogenesis of refractory wound and the application of artificial dermis to the treatment. Chinese Journal of Clinical Rational Drug Use. 2017; 10(28): 180-181.

[4] Junlin X, Hua Z, Yi H, et al. Application of improved vacuum sealing drainage technology and skin flap grafting in the treatment of deep chronic refractory wound. China Modern Medicine. 2014; 21(32): 25-27.

[5] Jixian M, Hongwei W, Feng G, et al. Analysis of Long-Term Effect of Limb Skin Flap Repair on Chronic Wound. The Journal of Medical Theory and Practice. 2015; 28(06): 721-722 + 725.
[6] Liming Y. Clinical observation of rhGM-CSF combined with alginate dressing in the treatment of surperficial chronic refractory wound in elderly patients. Shandong University. 2014.

[7] Zhidong R, Lingfeng W. Clinical observation on vacuum sealing technique for chronic wound. Chinese Journal of General Practice. 2013; 11(01): 29-31.

[8] Qiang L, Jiasong S. Chronic wound: pathogenesis and current treatments. Chinese Journal of New Clinical Medicine. 2013; 6(09): $917-$ 920

[9] Ling L, Guoping W. The effect of vacuum sealing drainage and nursing intervention on chronic and nonhealing wounds. Chinese Journal of Aesthetic Medicine. 2014; 23(01): 19-22.

[10] Haishui G, Yuan L, Haidong L, et al. Discussion on combined modality therapy of chronic refractory wound. Shanxi Medical Journal. 2014; 43(09): 1052-1054.

[11] Qian T, Yanan J. Application of skin flap in the treatment of chronic refractory wounds. Chinese Journal of Injury Repair and Wound Healing (Electronic Edition). 2017; 12(06): 414-420.

[12] Lan J. Retrospective analysis of VSD in the treatment of chronic refractory wound. Nanchang University. 2017. 